\title{
Ability of self-reported estimates of dietary sodium, potassium and protein to detect an association with general and abdominal obesity: comparison with the estimates derived from $24 \mathrm{~h}$ urinary excretion
}

\author{
Kentaro Murakami ${ }^{1,2 *}$, M. Barbara E. Livingstone ${ }^{3}$, Satoshi Sasaki ${ }^{2}$, Kazuhiro Uenishi ${ }^{4}$ and \\ the Japan Dietetic Students' Study for Nutrition and Biomarkers Group \\ ${ }^{1}$ Department of Nutrition, School of Human Cultures, University of Shiga Prefecture, Hikone, Shiga 522 8533, Japan \\ ${ }^{2}$ Department of Social and Preventive Epidemiology, School of Public Health, University of Tokyo, Tokyo, Japan \\ ${ }^{3}$ Northern Ireland Centre for Food and Health, University of Ulster, Coleraine, UK \\ ${ }^{4}$ Laboratory of Physiological Nutrition, Kagawa Nutrition University, Saitama, Japan
}

(Submitted 21 August 2014 - Final revision received 30 November 2014 - Accepted 28 January 2015 - First published online 18 March 2015)

\begin{abstract}
As under-reporting of dietary intake, particularly by overweight and obese subjects, is common in dietary surveys, biases inherent in the use of self-reported dietary information may distort true diet-obesity relationships or even create spurious ones. However, empirical evidence of this possibility is limited. The present cross-sectional study compared the relationships of $24 \mathrm{~h}$ urine-derived and self-reported intakes of $\mathrm{Na}, \mathrm{K}$ and protein with obesity. A total of 1043 Japanese women aged 18-22 years completed a $24 \mathrm{~h}$ urine collection and a selfadministered diet history questionnaire. After adjustment for potential confounders, $24 \mathrm{~h}$ urine-derived Na intake was associated with a higher risk of general obesity (BMI $\geq 25 \mathrm{~kg} / \mathrm{m}^{2}$ ) and abdominal obesity (waist circumference $\geq 80 \mathrm{~cm}$; both $P$ for trend=0.04). For $24 \mathrm{~h}$ urine-derived protein intake, positive associations with general and abdominal obesity were observed $(P$ for trend $=0.02$ and 0.053 , respectively). For $24 \mathrm{~h}$ urine-derived $\mathrm{K}$ intake, there was an inverse association with abdominal obesity $(P$ for trend=0.01). Conversely, when self-reported dietary information was used, only inverse associations between $\mathrm{K}$ intake and general and abdominal obesity were observed ( $P$ for trend $=0.04$ and 0.02 , respectively), with no associations of $\mathrm{Na}$ or protein intake. In conclusion, we found positive associations of $\mathrm{Na}$ and protein intakes and inverse associations of $\mathrm{K}$ intake with obesity when using $24 \mathrm{~h}$ urinary excretion for estimating dietary intakes. However, no association was observed based on using self-reported dietary intakes, except for inverse association of $\mathrm{K}$ intake, suggesting that the ability of self-reported dietary information using the diet history questionnaire for investigating diet-obesity relationships is limited.
\end{abstract}

Key words: 24 h Urine: Sodium: Protein: Obesity

Although accurate assessment of habitual dietary intake is a prerequisite in studies of diet and health, the difficulty of obtaining dietary data that accurately represents what people usually eat is now generally acknowledged ${ }^{(1)}$. Misreporting, particularly under-reporting, of energy intake, a surrogate measure of total food intake, by a variety of dietary assessment methods relative to total energy expenditure measured by the doubly labelled water method, the gold standard for measuring free-living total energy expenditure, is common ${ }^{(2,3)}$. Additionally, misreporting of energy intake within a population might not be random, but might rather occur systematically within certain groups of the population ${ }^{(1-4)}$. In particular, overweight and obese subjects tend to underreport energy intake to a greater extent than lean subjects ${ }^{(1-5)}$
Unfortunately, a potential solution to under-reporting, such as energy adjustment, is hindered by what appears to be a selective reporting of various nutrients and foods ${ }^{(4)}$. For example, protein is usually not under-reported to the same degree as carbohydrates and fats ${ }^{(2,4,6-8)}$, and between-meal snacks and foods considered to be unhealthy seem to be under-reported to a greater extent than those considered to be healthy ${ }^{(1,3,4,8)}$. Thus, biases inherent in the use of self-reported dietary information may distort or obscure the associations between diet and health, particularly obesity, or even create spurious ones. However, investigation of this possibility with the use of dietary biomarkers, such as $24 \mathrm{~h}$ urinary excretion of $\mathrm{N}$ (protein), $\mathrm{Na}$ and $\mathrm{K}^{(9-11)}$, is limited. In infants, for example, it has been found that a high intake of protein from

Abbreviations: DHQ, diet history questionnaire; PABA, para-aminobenzoic acid; WC, waist circumference.

*Corresponding author: Dr K. Murakami, fax +81 79449 8499, email kenmrkm@m.u-tokyo.ac.jp 
complementary feeding is associated with a higher risk of developing obesity, possibly because of the interplay with insulin-like growth factor- $1^{(12)}$. Such mechanisms could contribute to gain in fat mass with greater habitual protein intake in adults ${ }^{(13)}$. In a small study in middle-aged men and women in Denmark, both protein intake estimated from $24 \mathrm{~h}$ urinary $\mathrm{N}$ excretion and self-reported protein intake were associated with greater weight gain after 6 years ${ }^{(13)}$. However, the authors did not examine other nutrients including $\mathrm{Na}$ and $\mathrm{K}^{(13)}$, whose effect on obesity are now being investigated $^{(14-20)}$. For $\mathrm{Na}$, a mechanism not triggered by increased energy intake has been proposed by an animal study, where a high consumption of salt contributed to development of obesity among rats ${ }^{(21)}$. The increase in adipose tissue mass is suggested to be caused by an increased capacity to incorporate glucose into lipids, and a higher lipogenic enzymatic activity may have promoted adipocyte hypertrophy and then excessive fat accumulation ${ }^{(21)}$. A higher intake of $\mathrm{K}$ may also be associated with a lower risk of obesity mainly due to higher intakes of fruits and vegetables, the major sources of $\mathrm{K}^{(22)}$, although the effect of fruits and vegetables on obesity is controversial ${ }^{(23)}$. A very limited number of studies have shown associations between either self-reported or $24 \mathrm{~h}$ urine-derived intakes of $\mathrm{Na}$ and $\mathrm{K}$ with body fatness measures; however, no investigation has been done on the basis of using both self-reported and $24 \mathrm{~h}$ urine-derived estimates simultaneously ${ }^{(14-20)}$

Therefore, using $24 \mathrm{~h}$ urinary excretions of $\mathrm{Na}, \mathrm{K}$ and $\mathrm{N}$ (protein) as established quantitative biomarkers of intakes of these nutrients ${ }^{(9-11)}$, we compared the relationships of $24 \mathrm{~h}$ urine-derived and self-reported intakes of $\mathrm{Na}, \mathrm{K}$ and protein with general and abdominal obesity in a relatively large sample of young Japanese women. We hypothesised that the expected associations of $\mathrm{Na}, \mathrm{K}$ and protein intakes with obesity are observed only when using $24 \mathrm{~h}$ urinary excretion for estimating dietary intakes, but not based on using self-reported dietary intakes, because of obesity-related biases in self-reported dietary information.

\section{Subjects and methods}

\section{Study population}

The present cross-sectional study was based on a survey conducted from February to March 2006 and from January to March 2007 among female dietetic students from fifteen higher-education institutions in Japan. Details of the study design and survey procedure have been described elsewhere $^{(22,24,25)}$. Of the 1176 Japanese women who took part in the survey (response rate 56\%), 1105 conducted the $24 \mathrm{~h}$ urine collection. For the analysis, we selected women aged 18-22 years ( $n$ 1083). We then excluded those with missing information on the variables used ( $n$ 5). We further excluded those whose $24 \mathrm{~h}$ urine collection was considered incomplete ( $n$ 35), as assessed using the information on urinary creatinine excretion and body weight based on a strategy proposed by Knuiman et al. ${ }^{(26)}$. This creatinine-based strategy has been validated against the para-aminobenzoic acid (PABA) check method in a subsample ( $n$ 654) of the present subjects (\% of subjects having incomplete urine collection $5.5 \%$; sensitivity $0 \cdot 47$; specificity 0.99 ; \% of subjects misclassified $4 \%)^{(24)}$. Since exclusion of underweight subjects (BMI $<18.5 \mathrm{~kg} / \mathrm{m}^{2}$, $n$ 118) ${ }^{(27)}$ did not alter the findings of the present study (data not shown), these subjects were also included in the analysis, giving a final sample size of 1043 women.

The present study was conducted according to the guidelines laid down in the Declaration of Helsinki, and all procedures involving human subjects were approved by the ethics committee of the National Institute of Health and Nutrition, Japan. Written informed consent was obtained from each subject and also from a parent/guardian for subjects $<20$ years old.

\section{Anthropometric measurements}

Body height (to the nearest $0 \cdot 1 \mathrm{~cm}$ ) and weight (to the nearest $0.1 \mathrm{~kg}$ ) were measured while subjects were wearing lightweight indoor clothes only, without shoes. BMI $\left(\mathrm{kg} / \mathrm{m}^{2}\right)$ was calculated as weight (in $\mathrm{kg}$ ) divided by height (in $\mathrm{m}$ ) squared. Waist circumference (WC) was measured at the level of the umbilicus (to the nearest $0 \cdot 1 \mathrm{~cm}$ ) at the end of a normal respiration while the subject was standing erect and with the arms at the side and the feet together. General obesity was defined as BMI $\geq 25 \mathrm{~kg} / \mathrm{m}^{2}$ while abdominal obesity was defined as WC $\geq 80 \mathrm{~cm}$, based on cut-off points for Asian women according to the $\mathrm{WHO}^{(27)}$.

\section{4h Urine-derived dietary intake}

A single $24 \mathrm{~h}$ urine sample was collected from each subject. A detailed description of the $24 \mathrm{~h}$ urine collection procedure has been published elsewhere ${ }^{(22,24,25)}$. Briefly, subjects were asked to collect all urine voided during a $24 \mathrm{~h}$ period, and to record the time of the start and end of the collection period, and the estimated volume of any missing urine specimens. The $24 \mathrm{~h}$ urine volume was adjusted by selfreported collection time (calculated from the self-reported time of the start and end of the collection period) and missing urine volume. This adjustment strategy has been validated using the PABA check method in a subsample ( $n$ 654) of the present subjects ${ }^{(24)}$. All urine samples taken over the $24 \mathrm{~h}$ period were carefully mixed, and several aliquots were taken and transported at $-20^{\circ} \mathrm{C}$ to a laboratory (SRL, Inc. in 2006 and Mitsubishi Kagaku Bio-Clinical Laboratories, Inc. in 2007). In accordance with the standard procedure at each laboratory, urea $\mathrm{N}$ concentrations were measured using the enzymatic assay method, $\mathrm{Na}$ and $\mathrm{K}$ concentrations using the ion-selective electrode method and creatinine concentrations using the enzymatic assay method.

Total $24 \mathrm{~h}$ urinary excretion was calculated by multiplying the measured concentration by the (adjusted) volume of $24 \mathrm{~h}$ urine. To estimate $24 \mathrm{~h}$ urine-derived intake of protein, urea $\mathrm{N}$ content in urine was multiplied by $9 \cdot 08$, assuming that urea $\mathrm{N}$ is in constant proportion ( $85 \%)$ to total urinary $\mathrm{N}^{(9)}$, that $81 \%$ of ingested $\mathrm{N}$ is excreted through the urine ${ }^{(9)}$ and that $\mathrm{N}$ constitutes $16 \%$ of protein. The $24 \mathrm{~h}$ urine-derived 
intakes of $\mathrm{Na}$ and $\mathrm{K}$ were, respectively, estimated as $\mathrm{Na}$ content in urine divided by 0.86 (assuming that $86 \%$ of ingested $\mathrm{Na}$ is excreted through the urine $)^{(10)}$ and $\mathrm{K}$ content in urine divided by 0.77 (assuming that $77 \%$ of ingested $\mathrm{K}$ is excreted through the urine $)^{(11)}$. Energy adjustment based on the density method ( $\%$ of energy for protein and $\mathrm{mg} / 4184 \mathrm{~kJ}$ for $\mathrm{Na}$ and $\mathrm{K}$ ) was made using estimated energy requirement (i.e. total energy expenditure during weight stability), which was calculated with the use of sex- and age-specific equation published from the US Dietary Reference Intakes ${ }^{(28)}$, based on age, weight, height and self-reported physical activity ${ }^{(22)}$.

\section{Self-reported dietary intake}

Self-reported information on dietary habits during the preceding month was obtained using a comprehensive selfadministered diet history questionnaire $(\mathrm{DHQ})^{(29-32)}$, which was conducted $1-3 \mathrm{~d}$ before conducting $24 \mathrm{~h}$ urine collection and anthropometric measurements. Details of the structure of DHQ and calculation method of dietary intake have been published elsewhere ${ }^{(29-32)}$. Briefly, the DHQ is a structured sixteen-page questionnaire that asks about the consumption frequency and portion size of selected foods commonly consumed in Japan, as well as general dietary behaviour and usual cooking methods. Estimates of daily intake for foods (150 items in total), energy, protein, $\mathrm{Na}$ and $\mathrm{K}$ were calculated using an ad hoc computer algorithm for the DHQ based on the Standard Tables of Food Composition in Japan ${ }^{(33)}$. Energy adjustment was made using self-reported energy intake based on the density method. Validity on the DHQ with respect to commonly studied nutritional factors has been investigated in several previous studies ${ }^{(29-32)}$. For example, Pearson's correlation coefficients were 0.52 for protein, 0.53 for $\mathrm{K}$ and 0.39 for $\mathrm{Na}$ between the DHQ and 16- $\mathrm{d}$ weighed dietary record in ninety-two women aged $31-69$ years $^{(31)}$, and 0.40 for $\mathrm{K}$ and 0.23 for $\mathrm{Na}$ between the DHQ and $24 \mathrm{~h}$ urinary excretion in sixty-nine female college students ${ }^{(29)}$. Further, Pearson's correlation coefficient between energy intake derived from the DHQ and total energy expenditure measured by the doubly labelled water was $0 \cdot 22$ in seventy-three women aged $20-59$ years $^{(30)}$

\section{Other variables}

Based on the reported home address, each subject was grouped into one of the three regions (north (Kanto, Hokkaido and Tohoku); central (Tokai, Hokuriku and Kinki); or south (Kyushu and Chugoku)) and into one of the three municipality levels (ward (i.e. metropolitan area), city or town and village). Residential status (living with family, living alone or living with others), current alcohol drinking (yes or no) and current smoking status (yes or no) were also self-reported. Physical activity was computed as the average metabolic equivalent hours score per day on the basis of the self-reported frequency and duration of five activities (sleeping, high- and moderate-intensity activities, walking and sedentary activities) over the preceding month $^{(22)}$.

\section{Statistical analyses}

All statistical analyses were performed using SAS statistical software (version 9.2; SAS Institute, Inc.). The $24 \mathrm{~h}$ urine-derived and self-reported intakes of $\mathrm{Na}, \mathrm{K}$ and protein were categorised at quartile points based on the distribution. With the use of the PROC GLM procedure, multivariate-adjusted means (with standard errors) of BMI and WC were calculated according to the quartiles of dietary intakes. Further, using the PROC LOGISTIC procedure, multivariate-adjusted OR (95\% CI) for general and abdominal obesity were calculated for each quartiles of dietary intakes, with the lowest quartile category used as the reference. We tested for linear trends with increasing levels of dietary intakes by assigning each subject the median value for the category and modelling this value as a continuous variable. The potential confounding factors considered were survey year, region, municipality level, residential status, current alcohol drinking, current smoking status, physical activity and $24 \mathrm{~h}$ urine-derived or self-reported intakes of other nutrients. All reported $P$ values are two-tailed, and $P$ values $<0.05$ were considered statistically significant.

\section{Results}

The mean values of BMI and WC were $21.2 \mathrm{~kg} / \mathrm{m}^{2}$ and $72.7 \mathrm{~cm}$, respectively (Table 1 ). The mean daily values of $24 \mathrm{~h}$ urine-derived and self-reported dietary intakes were 1962 and $2055 \mathrm{mg} / 4184 \mathrm{~kJ}$ for $\mathrm{Na}, 1144$ and $1114 \mathrm{mg} / 4184 \mathrm{~kJ}$ for $\mathrm{K}$ and 14.0 and $13.5 \%$ of energy from protein, respectively. Pearson's correlation coefficients between $24 \mathrm{~h}$ urine-derived and self-reported intakes were 0.08 for $\mathrm{Na}, 0.28$ for $\mathrm{K}$ and 0.20 for protein. The prevalence of general and abdominal obesity was 7.7 and $13.0 \%$, respectively. Subjects with general obesity were less likely to live alone and more likely to live with others. There were more subjects defined as abdominally obese in the regions of north and south, with fewer in the region of central. No difference in self-reported energy intake was observed between obese (general or abdominal) subjects and their non-obese counterparts. Both general and abdominal obese subjects had a higher mean value of estimated energy requirement and $24 \mathrm{~h}$ urine-derived $\mathrm{Na}$ intake. Abdominal obese subjects also had a lower mean value of self-reported intakes of all the three nutrients.

There were positive associations among $24 \mathrm{~h}$ urine-derived intakes of all the three nutrients (Pearson's correlation coefficient: 0.35 for $\mathrm{Na}$ and $\mathrm{K} ; 0.47$ for $\mathrm{Na}$ and protein; 0.55 for $\mathrm{K}$ and protein; Table 2). Additionally, $24 \mathrm{~h}$ urine-derived intakes of all the three nutrients were associated with survey year, region and residential status. The higher quartile of intakes included more subjects in the 2007 survey; more subjects in the region of central and fewer in the region of north (except $\mathrm{K}$ ); and more subjects living with family and fewer living alone (protein only). The $24 \mathrm{~h}$ urine-derived intakes also showed a positive association with current alcohol drinking and inverse associations with current smoking status (protein only) and physical activity.

As was the case in $24 \mathrm{~h}$ urine-derived intakes, there were positive associations between self-reported intakes of all the 
Table 1. Basic characteristics of subjects*

(Mean values and standard deviations or percentages)

\begin{tabular}{|c|c|c|c|c|c|c|c|c|c|c|c|c|}
\hline & & & \multicolumn{4}{|c|}{ General obesity } & \multicolumn{5}{|c|}{ Abdominal obesity } & \multirow[b]{3}{*}{$P \dagger$} \\
\hline & \multicolumn{2}{|c|}{ All $(n$ 1043) } & \multicolumn{2}{|c|}{$\begin{array}{l}\text { Yes }(n 80 \\
7.7 \%)\end{array}$} & \multicolumn{2}{|c|}{$\begin{array}{l}\text { No }(n 963 \\
92 \cdot 3 \%)\end{array}$} & \multirow[b]{2}{*}{$P \dagger$} & \multicolumn{2}{|c|}{$\begin{array}{l}\text { Yes }(n 136 \\
13.0 \%)\end{array}$} & \multicolumn{2}{|c|}{$\begin{array}{l}\text { No }(n 907 \\
87.0 \%)\end{array}$} & \\
\hline & Mean & SD & Mean & SD & Mean & SD & & Mean & SD & Mean & SD & \\
\hline Age (years) & 19.6 & 1.0 & 19.6 & $1 \cdot 1$ & 19.6 & 1.0 & 0.73 & $19 \cdot 4$ & 1.0 & $19 \cdot 6$ & 1.0 & 0.01 \\
\hline Body height $(\mathrm{cm})$ & 158.4 & 5.4 & 158.8 & $5 \cdot 5$ & 158.3 & 5.4 & 0.52 & $160 \cdot 2$ & $5 \cdot 1$ & $158 \cdot 1$ & $5 \cdot 4$ & $<0.0001$ \\
\hline Body weight (kg) & $53 \cdot 3$ & $7 \cdot 3$ & $68 \cdot 2$ & 7.4 & $52 \cdot 0$ & $5 \cdot 7$ & $<0.0001$ & $64 \cdot 2$ & $8 \cdot 1$ & 51.6 & 5.5 & $<0.0001$ \\
\hline BMI $\left(\mathrm{kg} / \mathrm{m}^{2}\right)$ & $21 \cdot 2$ & $2 \cdot 5$ & $27 \cdot 0$ & 1.9 & $20 \cdot 7$ & 1.9 & $<0.0001$ & $25 \cdot 0$ & $2 \cdot 7$ & $20 \cdot 7$ & 1.9 & $<0.0001$ \\
\hline Waist circumference $(\mathrm{cm})$ & $72 \cdot 7$ & $6 \cdot 8$ & $85 \cdot 2$ & $6 \cdot 8$ & $71 \cdot 7$ & $5 \cdot 7$ & $<0.0001$ & $85 \cdot 0$ & $5 \cdot 0$ & $70 \cdot 9$ & 4.9 & $<0.0001$ \\
\hline Survey year (\%) & \multirow{2}{*}{\multicolumn{2}{|c|}{38.6}} & & & & & 0.49 & & & & & $0 \cdot 16$ \\
\hline 2006 & & & \multicolumn{2}{|c|}{$35 \cdot 0$} & \multicolumn{2}{|c|}{38.9} & & \multicolumn{2}{|c|}{$44 \cdot 1$} & \multicolumn{2}{|c|}{37.8} & \\
\hline 2007 & \multicolumn{2}{|c|}{61.4} & \multicolumn{2}{|c|}{$65 \cdot 0$} & \multicolumn{2}{|c|}{$61 \cdot 1$} & & \multicolumn{2}{|c|}{$55 \cdot 9$} & \multicolumn{2}{|c|}{$62 \cdot 2$} & \\
\hline Region (\%) & & & & & & & 0.45 & & & & & 0.01 \\
\hline North & \multicolumn{2}{|c|}{57.9} & \multicolumn{2}{|c|}{$60 \cdot 0$} & \multicolumn{2}{|c|}{57.7} & & \multicolumn{2}{|c|}{64.7} & \multicolumn{2}{|c|}{$56 \cdot 9$} & \\
\hline Central & \multicolumn{2}{|c|}{$24 \cdot 1$} & & & 24 & & & & & & & \\
\hline South & 18 & & & & 17 & & & & & & & \\
\hline Municipality level (\%) & & & & & & & 0.70 & & & & & 0.47 \\
\hline Ward & 16 & & & & 16 & & & & & 16 & & \\
\hline City & $7 \varepsilon$ & & & & $7 \varepsilon$ & & & & & & & \\
\hline Town and village & & & & & 5 & & & 5 & & 5 & & \\
\hline Residential status (\%) & & & & & & & 0.002 & & & & & 0.18 \\
\hline Living with family & 60 & & & & 60 & & & & & & & \\
\hline Living alone & 35 & & 27 & & 36 & & & & & & & \\
\hline Living with others & 4 & & 11 & & & & & & & & & \\
\hline Current alcohol drinking (\%) & & & & & & & $0 \cdot 16$ & & & & & $0 \cdot 10$ \\
\hline Current smoking (\%) & & & & & & & 0.14 & & & & & 0.41 \\
\hline $\begin{array}{l}\text { Physical activity } \\
\text { (total metabolic equivalents-h/d) }\end{array}$ & 33.9 & $3 \cdot 1$ & $34 \cdot 3$ & $5 \cdot 3$ & 33.9 & $2 \cdot 8$ & 0.34 & 34.0 & 4.6 & 33.9 & $2 \cdot 8$ & 0.73 \\
\hline Self-reported energy intake $(\mathrm{kJ} / \mathrm{d})$ & 7414 & 1896 & 7585 & 1889 & 7400 & 1897 & 0.40 & 7503 & 1920 & 7401 & 1893 & 0.56 \\
\hline Estimated energy requirement $(\mathrm{kJ} / \mathrm{d})$ & 8275 & 766 & 8942 & 941 & 8220 & 723 & $<0.0001$ & 8826 & 862 & 8193 & 715 & $<0.0001$ \\
\hline $24 \mathrm{~h}$ Urine-derived dietary intake $\ddagger$ & & & & & & & & & & & & \\
\hline $\mathrm{Na}(\mathrm{mg} / 4184 \mathrm{~kJ})$ & 1962 & 751 & 2143 & 728 & 1947 & 752 & 0.03 & 2087 & 782 & 1944 & 745 & 0.04 \\
\hline $\mathrm{K}(\mathrm{mg} / 4184 \mathrm{~kJ})$ & 1144 & 413 & 1103 & 337 & 1148 & 419 & 0.36 & 1116 & 416 & 1148 & 413 & 0.40 \\
\hline Protein (\% of energy) & 14.0 & 3.5 & 14.5 & $3 \cdot 1$ & 13.9 & 3.5 & 0.15 & $14 \cdot 3$ & $3 \cdot 7$ & 13.9 & 3.5 & 0.21 \\
\hline Self-reported dietary intake§ & & & & & & & & & & & & \\
\hline $\mathrm{Na}(\mathrm{mg} / 4184 \mathrm{~kJ})$ & 2055 & 492 & 2036 & 503 & 2056 & 491 & 0.73 & 1955 & 522 & 2069 & 485 & 0.01 \\
\hline $\mathrm{K}(\mathrm{mg} / 4184 \mathrm{~kJ})$ & 1114 & 257 & 1067 & 288 & 1118 & 254 & 0.09 & 1038 & 256 & 1126 & 255 & 0.0002 \\
\hline Protein (\% of energy) & $13 \cdot 5$ & 1.9 & $13 \cdot 2$ & $2 \cdot 0$ & $13 \cdot 5$ & $1 \cdot 8$ & 0.14 & $13 \cdot 0$ & 1.8 & 13.6 & 1.9 & 0.0004 \\
\hline
\end{tabular}

${ }^{*}$ General obesity was defined as $\mathrm{BMI} \geq 25 \mathrm{~kg} / \mathrm{m}^{2}$; abdominal obesity was defined as waist circumference $\geq 80 \mathrm{~cm}^{(27)}$.

$\dagger P$ values for differences between (generally or centrally) obese and non-obese subjects based on the independent $t$ test for continuous variables and the $\chi^{2}$ test for categorical variables.

$\ddagger$ Energy adjustment was made using estimated energy requirement.

$\S$ Energy adjustment was made using self-reported energy intake.

three nutrients (Pearson's correlation coefficient: 0.31 for $\mathrm{Na}$ and $\mathrm{K} ; 0.41$ for $\mathrm{Na}$ and protein; 0.63 for $\mathrm{K}$ and protein; Table 3). Further, self-reported intakes of all the three nutrients were associated with survey year, region, municipality level and residential status. The higher quartiles of intakes included more subjects in the 2006 survey; more subjects in the region of north and fewer in the region of south; more subjects living in wards and fewer living in towns and villages (protein only); and more subjects living with family and fewer living alone. Self-reported protein intake was also inversely associated with current smoking status.

After adjustment for potential confounding variables, $24 \mathrm{~h}$ urine-derived $\mathrm{Na}$ intake was positively associated with BMI ( $P$ for trend $=0.005$ ) but not with WC (Table 4 ). The $24 \mathrm{~h}$ urine-derived protein intake also showed independent positive associations with both BMI ( $P$ for trend=0.0008) and WC ( $P$ for trend=0.02). Conversely, $24 \mathrm{~h}$ urine-derived $\mathrm{K}$ intake was independently and inversely associated with both BMI ( $P$ for trend=0.03) and WC ( $P$ for trend=0.04). When self-reported dietary intakes were examined, intakes of $\mathrm{Na}$ and protein were not associated with BMI or WC. However, self-reported $\mathrm{K}$ intake showed independent and inverse associations with BMI $(P$ for trend $=0.009)$ and $\mathrm{WC}$ ( $P$ for trend $=0.001$ ).

Independent associations of $\mathrm{Na}$, K and protein intakes with general and abdominal obesity are summarised in Table 5 . The $24 \mathrm{~h}$ urine-derived $\mathrm{Na}$ intake was associated with a higher risk of both general (adjusted OR between extreme quartiles 2.49; $95 \%$ CI $1 \cdot 15,5 \cdot 42 ; P$ for trend $=0.04$ ) and abdominal (adjusted OR $1 \cdot 77 ; 95 \%$ CI 1.00, 3.16; $P$ for trend=0.04) obesity. Similarly, $24 \mathrm{~h}$ urine-derived protein intake showed positive associations with both general (adjusted OR 2.95; 95\% CI 1.21, 7.22; $P$ for trend=0.02) and abdominal (adjusted OR 1.97; 95\% CI 1.02, 3.83; $P$ for trend $=0.053$ ) obesity. Conversely, $24 \mathrm{~h}$ urine-derived $\mathrm{K}$ intake was inversely associated with abdominal obesity only (adjusted OR 0.46; 95\% CI 0.25, 0.87; $P$ for trend=0.01). 
Table 2. Selected characteristics of subjects according to the quartiles $(\mathrm{Q})$ of $24 \mathrm{~h}$ urine-derived dietary intakes of sodium, potassium and protein $(n 1043)$ (Mean values or percentages)

\begin{tabular}{|c|c|c|c|c|c|c|c|c|c|c|c|c|c|c|c|}
\hline & \multicolumn{4}{|c|}{$24 \mathrm{~h}$ Urine-derived $\mathrm{Na}$ intake } & \multirow{3}{*}{$\begin{array}{l}P \text { for } \\
\text { trend }^{*}\end{array}$} & \multicolumn{4}{|c|}{ 24h Urine-derived $\mathrm{K}$ intake } & \multirow{3}{*}{$\begin{array}{l}P \text { for } \\
\text { trend }\end{array}$} & \multicolumn{4}{|c|}{$24 \mathrm{~h}$ Urine-derived protein intake } & \multirow{3}{*}{$\begin{array}{l}P \text { for } \\
\text { trend* }\end{array}$} \\
\hline & $\begin{array}{c}\mathrm{Q} 1 \\
(n 260)\end{array}$ & $\begin{array}{c}\text { Q2 } \\
(n 261)\end{array}$ & $\begin{array}{c}\text { Q3 } \\
(n 261)\end{array}$ & $\begin{array}{c}\mathrm{Q} 4 \\
(n 261)\end{array}$ & & $\begin{array}{c}\mathrm{Q} 1 \\
(n 260)\end{array}$ & $\begin{array}{c}\mathrm{Q} 2 \\
(n 261)\end{array}$ & $\begin{array}{c}\text { Q3 } \\
(n 261)\end{array}$ & $\begin{array}{c}\text { Q4 } \\
(n 261)\end{array}$ & & $\begin{array}{c}\text { Q1 } \\
(n 260)\end{array}$ & $\begin{array}{c}\mathrm{Q} 2 \\
(n 261)\end{array}$ & $\begin{array}{c}\text { Q3 } \\
(n 261)\end{array}$ & $\begin{array}{c}\text { Q4 } \\
(n 261)\end{array}$ & \\
\hline & Mean & Mean & Mean & Mean & & Mean & Mean & Mean & Mean & & Mean & Mean & Mean & Mean & \\
\hline Survey year (\%) & & & & & $<0.0001$ & & & & & $<0.0001$ & & & & & $<0.0001$ \\
\hline 2006 & $49 \cdot 6$ & $38 \cdot 3$ & 36.4 & $30 \cdot 3$ & & $46 \cdot 9$ & $44 \cdot 1$ & $33 \cdot 7$ & $29 \cdot 9$ & & $51 \cdot 2$ & $42 \cdot 5$ & 33.0 & 28.0 & \\
\hline 2007 & $50 \cdot 4$ & $61 \cdot 7$ & $63 \cdot 6$ & $69 \cdot 7$ & & $53 \cdot 1$ & 55.9 & $66 \cdot 3$ & $70 \cdot 1$ & & $48 \cdot 9$ & 57.5 & $67 \cdot 1$ & $72 \cdot 0$ & \\
\hline Region (\%) & & & & & 0.004 & & & & & 0.06 & & & & & 0.0004 \\
\hline North & $66 \cdot 2$ & $60 \cdot 9$ & 53.6 & $51 \cdot 0$ & & $59 \cdot 6$ & 60.5 & $59 \cdot 0$ & 52.5 & & $65 \cdot 8$ & $62 \cdot 8$ & $60 \cdot 2$ & $42 \cdot 9$ & \\
\hline Central & $16 \cdot 5$ & $23 \cdot 8$ & $26 \cdot 1$ & 29.9 & & $22 \cdot 7$ & 24.9 & $22 \cdot 6$ & $26 \cdot 1$ & & $15 \cdot 4$ & $20 \cdot 7$ & $21 \cdot 8$ & $38 \cdot 3$ & \\
\hline South & $17 \cdot 3$ & $15 \cdot 3$ & $20 \cdot 3$ & $19 \cdot 2$ & & $17 \cdot 7$ & $14 \cdot 6$ & $18 \cdot 4$ & 21.5 & & $18 \cdot 9$ & $16 \cdot 5$ & $18 \cdot 0$ & $18 \cdot 8$ & \\
\hline Municipality level (\%) & & & & & 0.31 & & & & & 0.35 & & & & & 0.36 \\
\hline Ward & $16 \cdot 9$ & 14.9 & $15 \cdot 7$ & $16 \cdot 9$ & & $17 \cdot 7$ & 13.4 & $18 \cdot 0$ & $15 \cdot 3$ & & $13 \cdot 9$ & $21 \cdot 1$ & 14.6 & 14.9 & \\
\hline City & $79 \cdot 2$ & $80 \cdot 1$ & $78 \cdot 2$ & $75 \cdot 1$ & & $77 \cdot 3$ & $82 \cdot 0$ & $75 \cdot 9$ & $77 \cdot 4$ & & $82 \cdot 3$ & 72.4 & $79 \cdot 7$ & $78 \cdot 2$ & \\
\hline Town and village & 3.9 & $5 \cdot 0$ & $6 \cdot 1$ & $8 \cdot 1$ & & $5 \cdot 0$ & 4.6 & $6 \cdot 1$ & $7 \cdot 3$ & & 3.9 & 6.5 & $5 \cdot 8$ & 6.9 & \\
\hline Residential status (\%) & & & & & 0.07 & & & & & 0.06 & & & & & $<0.0001$ \\
\hline Living with family & 53.9 & $60 \cdot 5$ & $64 \cdot 8$ & $62 \cdot 8$ & & $54 \cdot 2$ & 57.9 & $63 \cdot 6$ & $66 \cdot 3$ & & $47 \cdot 7$ & $63 \cdot 6$ & $61 \cdot 3$ & $69 \cdot 4$ & \\
\hline Living alone & 41.9 & $35 \cdot 6$ & 31.4 & $32 \cdot 6$ & & $42 \cdot 7$ & $38 \cdot 3$ & $33 \cdot 3$ & $27 \cdot 2$ & & $46 \cdot 9$ & $33 \cdot 3$ & 34.9 & $26 \cdot 4$ & \\
\hline Living with others & $4 \cdot 2$ & 3.8 & 3.8 & 4.6 & & $3 \cdot 1$ & $3 \cdot 8$ & $3 \cdot 1$ & 6.5 & & 5.4 & $3 \cdot 1$ & 3.8 & $4 \cdot 2$ & \\
\hline $\begin{array}{l}\text { Current alcohol } \\
\text { drinking (\%) }\end{array}$ & $37 \cdot 3$ & $42 \cdot 9$ & 41.8 & 47.9 & 0.02 & $36 \cdot 2$ & $38 \cdot 7$ & $47 \cdot 1$ & 47.9 & 0.002 & $37 \cdot 7$ & 41.4 & $44 \cdot 8$ & $46 \cdot 0$ & 0.04 \\
\hline Current smoking status (\%) & $2 \cdot 7$ & 1.5 & 2.7 & $3 \cdot 1$ & 0.59 & $3 \cdot 1$ & $2 \cdot 3$ & 1.9 & 2.7 & 0.80 & 3.9 & $2 \cdot 7$ & $2 \cdot 3$ & 1.2 & 0.049 \\
\hline $\begin{array}{l}\text { Physical activity } \\
\text { (total metabolic } \\
\text { equivalents-h/d) }\end{array}$ & $34 \cdot 5$ & $34 \cdot 2$ & $33 \cdot 6$ & 33.5 & $<0.0001$ & $34 \cdot 6$ & $34 \cdot 0$ & $33 \cdot 5$ & $33 \cdot 7$ & 0.0005 & $34 \cdot 7$ & $34 \cdot 1$ & $33 \cdot 6$ & $33 \cdot 3$ & $<0.0001$ \\
\hline $\begin{array}{c}24 \mathrm{~h} \text { Urine-derived } \\
\text { dietary intake† }\end{array}$ & & & & & & & & & & & & & & & \\
\hline $\mathrm{Na}(\mathrm{mg} / 4184 \mathrm{~kJ})$ & 1109 & 1662 & 2119 & 2957 & $<0.0001$ & 1652 & 1839 & 2025 & 2332 & $<0.0001$ & 1561 & 1770 & 2105 & 2413 & $<0.0001$ \\
\hline $\mathrm{K}(\mathrm{mg} / 4184 \mathrm{~kJ})$ & 984 & 1083 & 1166 & 1343 & $<0.0001$ & 694 & 965 & 1203 & 1712 & $<0.0001$ & 870 & 1049 & 1207 & 1450 & $<0.0001$ \\
\hline Protein (\% of energy) & $12 \cdot 0$ & $13 \cdot 4$ & $14 \cdot 2$ & $16 \cdot 3$ & $<0.0001$ & 11.5 & $13 \cdot 2$ & $14 \cdot 7$ & $16 \cdot 5$ & $<0.0001$ & $10 \cdot 0$ & $12 \cdot 6$ & $14 \cdot 6$ & $18 \cdot 6$ & $<0.0001$ \\
\hline
\end{tabular}

${ }^{*}$ For categorical variables, a Mantel-Haenszel $\chi^{2}$ test was used; for continuous variables, a linear trend test was used with the median value in each quartile category of dietary intake as a continuous variable in linear regression. $\dagger$ Energy adjustment was made using estimated energy requirement. 
Table 3. Selected characteristics of subjects according to the quartiles $(Q)$ of self-reported dietary intakes of sodium, potassium and protein ( $n$ 1043) (Mean values or percentages)

\begin{tabular}{|c|c|c|c|c|c|c|c|c|c|c|c|c|c|c|c|}
\hline & \multicolumn{4}{|c|}{ Self-reported $\mathrm{Na}$ intake } & \multirow{3}{*}{$\begin{array}{l}P \text { for } \\
\text { trend }\end{array}$} & \multicolumn{4}{|c|}{ Self-reported K intake } & \multicolumn{5}{|c|}{ Self-reported protein intake } & \multirow{3}{*}{$\begin{array}{l}P \text { for } \\
\text { trend* }\end{array}$} \\
\hline & $\begin{array}{c}\text { Q1 } \\
(n 260)\end{array}$ & $\begin{array}{c}\mathrm{Q} 2 \\
(n 261)\end{array}$ & $\begin{array}{c}\text { Q3 } \\
(n 261)\end{array}$ & $\begin{array}{c}\mathrm{Q} 4 \\
(n 261)\end{array}$ & & $\begin{array}{c}\mathrm{Q1} \\
(n 260)\end{array}$ & $\begin{array}{c}\mathrm{Q} 2 \\
(n 261)\end{array}$ & $\begin{array}{c}\text { Q3 } \\
(n 261)\end{array}$ & $\begin{array}{c}\mathrm{Q} 4 \\
(n 261)\end{array}$ & \multirow{2}{*}{$\begin{array}{l}P \text { for } \\
\text { trend }^{*}\end{array}$} & $\begin{array}{c}\mathrm{Q1} \\
(n 260)\end{array}$ & $\begin{array}{c}\text { Q2 } \\
(n 261)\end{array}$ & $\begin{array}{c}\text { Q3 } \\
(n \text { 261) }\end{array}$ & $\begin{array}{c}\text { Q4 } \\
(n 261)\end{array}$ & \\
\hline & Mean & Mean & Mean & Mean & & Mean & Mean & Mean & Mean & & Mean & Mean & Mean & Mean & \\
\hline Survey year (\%) & & & & & 0.0007 & & & & & \multirow[t]{3}{*}{0.009} & & & & & \multirow[t]{3}{*}{$<0.0001$} \\
\hline 2006 & 31.9 & $36 \cdot 0$ & 41.0 & $45 \cdot 6$ & & 31.9 & $35 \cdot 6$ & $45 \cdot 2$ & 41.8 & & 28.9 & $35 \cdot 3$ & $43 \cdot 7$ & $46 \cdot 7$ & \\
\hline 2007 & $68 \cdot 1$ & 64.0 & $59 \cdot 0$ & 54.4 & & 68.1 & 64.4 & 54.8 & $58 \cdot 2$ & & $71 \cdot 2$ & 64.8 & $56 \cdot 3$ & $53 \cdot 3$ & \\
\hline Region (\%) & & & & & 0.02 & & & & & \multirow[t]{4}{*}{0.0497} & & & & & \multirow[t]{4}{*}{0.0009} \\
\hline North & 51.5 & $58 \cdot 6$ & 59.8 & $61 \cdot 7$ & & $55 \cdot 8$ & $55 \cdot 6$ & 57.9 & 62.5 & & 51.5 & $56 \cdot 7$ & $58 \cdot 6$ & 64.8 & \\
\hline Central & $27 \cdot 3$ & $21 \cdot 1$ & $26 \cdot 1$ & $21 \cdot 8$ & & 23.9 & 24.9 & 24.9 & $22 \cdot 6$ & & $25 \cdot 4$ & 24.9 & 24.5 & 21.5 & \\
\hline South & $21 \cdot 2$ & $20 \cdot 3$ & $14 \cdot 2$ & $16 \cdot 5$ & & $20 \cdot 4$ & 19.5 & $17 \cdot 2$ & 14.9 & & $23 \cdot 1$ & $18 \cdot 4$ & $16 \cdot 9$ & $13 \cdot 8$ & \\
\hline Municipality level (\%) & & & & & 0.34 & & & & & \multirow[t]{4}{*}{0.78} & & & & & \multirow[t]{4}{*}{0.007} \\
\hline Ward & $14 \cdot 2$ & $15 \cdot 7$ & $15 \cdot 3$ & $19 \cdot 2$ & & $13 \cdot 1$ & $17 \cdot 2$ & $16 \cdot 9$ & $17 \cdot 2$ & & $10 \cdot 8$ & $12 \cdot 6$ & $22 \cdot 6$ & 18.4 & \\
\hline City & $80 \cdot 4$ & 78.9 & 78.5 & 74.7 & & $83 \cdot 1$ & 77.4 & 75.5 & $76 \cdot 6$ & & 83.5 & $81 \cdot 6$ & $70 \cdot 1$ & $77 \cdot 4$ & \\
\hline Town and village & $2 \cdot 4$ & $2 \cdot 4$ & $6 \cdot 1$ & $6 \cdot 1$ & & 3.9 & 5.4 & $7 \cdot 7$ & $6 \cdot 1$ & & $5 \cdot 8$ & $5 \cdot 8$ & $7 \cdot 3$ & $4 \cdot 2$ & \\
\hline Residential status (\%) & & & & & 0.0001 & & & & & \multirow[t]{4}{*}{$<0.0001$} & & & & & \multirow[t]{4}{*}{$<0.0001$} \\
\hline Living with family & $52 \cdot 3$ & $59 \cdot 0$ & $62 \cdot 1$ & 68.6 & & $44 \cdot 6$ & $59 \cdot 4$ & 65.9 & $72 \cdot 0$ & & $40 \cdot 8$ & $61 \cdot 7$ & $67 \cdot 1$ & $72 \cdot 4$ & \\
\hline Living alone & $40 \cdot 4$ & $37 \cdot 6$ & $36 \cdot 8$ & $26 \cdot 8$ & & 49.6 & $36 \cdot 4$ & $30 \cdot 7$ & 24.9 & & $52 \cdot 3$ & $36 \cdot 4$ & $28 \cdot 7$ & $24 \cdot 1$ & \\
\hline Living with others & $7 \cdot 3$ & 3.5 & $1 \cdot 2$ & 4.6 & & $5 \cdot 8$ & $4 \cdot 2$ & 3.5 & $3 \cdot 1$ & & 6.9 & 1.9 & 4.2 & 3.5 & \\
\hline $\begin{array}{l}\text { Current alcohol } \\
\text { drinking }(\%)\end{array}$ & $45 \cdot 4$ & $42 \cdot 2$ & $42 \cdot 2$ & $40 \cdot 2$ & 0.26 & 43.5 & $43 \cdot 7$ & $40 \cdot 6$ & $42 \cdot 2$ & 0.66 & $45 \cdot 4$ & $42 \cdot 2$ & $37 \cdot 2$ & $45 \cdot 2$ & 0.87 \\
\hline Current smoking status (\%) & 3.5 & $1 \cdot 2$ & 1.9 & 3.5 & 0.79 & 4.2 & 1.2 & $2 \cdot 3$ & $2 \cdot 3$ & 0.34 & 5.8 & $2 \cdot 3$ & 1.2 & 0.8 & 0.0003 \\
\hline $\begin{array}{l}\text { Physical activity } \\
\text { (total metabolic } \\
\text { equivalents-h/d) }\end{array}$ & $33 \cdot 8$ & $34 \cdot 0$ & 33.9 & $34 \cdot 1$ & 0.25 & $33 \cdot 8$ & 33.9 & $33 \cdot 9$ & $34 \cdot 2$ & $0 \cdot 14$ & $34 \cdot 1$ & $33 \cdot 7$ & $33 \cdot 9$ & $34 \cdot 1$ & 0.81 \\
\hline \multicolumn{16}{|l|}{$\begin{array}{l}\text { Self-reported dietary } \\
\text { intake† }\end{array}$} \\
\hline $\mathrm{Na}(\mathrm{mg} / 4184 \mathrm{~kJ})$ & 1475 & 1883 & 2171 & 2686 & $<0.0001$ & 1862 & 2016 & 2066 & 2274 & $<0.0001$ & 1808 & 1988 & 2115 & 2307 & $<0.0001$ \\
\hline $\mathrm{K}(\mathrm{mg} / 4184 \mathrm{~kJ})$ & 1001 & 1086 & 1137 & 1232 & $<0.0001$ & 818 & 1015 & 1171 & 1452 & $<0.0001$ & 927 & 1045 & 1160 & 1325 & $<0.0001$ \\
\hline Protein (\% of energy) & $12 \cdot 5$ & $13 \cdot 3$ & $13 \cdot 8$ & 14.5 & $<0.0001$ & $12 \cdot 0$ & $13 \cdot 2$ & 13.9 & $15 \cdot 0$ & $<0.0001$ & $11 \cdot 3$ & $12 \cdot 9$ & $14 \cdot 0$ & $15 \cdot 9$ & $<0.0001$ \\
\hline
\end{tabular}

${ }^{*}$ For categorical variables, a Mantel-Haenszel $\chi^{2}$ test was used; for continuous variables, a linear trend test was used with the median value in each quartile category of dietary intake as a continuous variable in linear regression. †Energy adjustment was made using self-reported energy intake. 
Table 4. BMI and waist circumference according to the quartiles (Q) of sodium, potassium and protein intakes $(n 1043)$

(Mean values with their standard errors)

\begin{tabular}{|c|c|c|c|c|c|c|c|c|c|}
\hline & \multicolumn{2}{|c|}{ Q1 (n 260) } & \multicolumn{2}{|c|}{ Q2 (n 261) } & \multicolumn{2}{|c|}{ Q3 (n 261) } & \multicolumn{2}{|c|}{ Q4 (n 261) } & \multirow[b]{2}{*}{$P$ for trend } \\
\hline & Mean & SE & Mean & SE & Mean & SE & Mean & SE & \\
\hline \multicolumn{10}{|l|}{$24 \mathrm{~h}$ Urine-derived dietary intake $†$} \\
\hline $\mathrm{Na}$ (mg/4184 kJ; median) & \multicolumn{2}{|c|}{1155} & \multicolumn{2}{|c|}{1659} & \multicolumn{2}{|c|}{2124} & \multicolumn{2}{|c|}{2766} & \\
\hline BMI $\left(\mathrm{kg} / \mathrm{m}^{2}\right)$ & & & & & & & & & \\
\hline Crude & $21 \cdot 0$ & 0.2 & $21 \cdot 2$ & 0.2 & $21 \cdot 1$ & 0.2 & $21 \cdot 7$ & 0.2 & 0.001 \\
\hline Adjusted $\ddagger$ & $21 \cdot 0$ & 0.2 & $21 \cdot 2$ & 0.2 & $21 \cdot 1$ & 0.2 & $21 \cdot 7$ & 0.2 & 0.005 \\
\hline \multicolumn{10}{|l|}{ Waist circumference $(\mathrm{cm})$} \\
\hline Crude & $72 \cdot 7$ & 0.4 & $72 \cdot 6$ & 0.4 & 72.5 & 0.4 & 73.1 & 0.4 & 0.48 \\
\hline Adjusted $\neq$ & $72 \cdot 3$ & 0.4 & $72 \cdot 6$ & 0.4 & $72 \cdot 6$ & 0.4 & 73.3 & 0.4 & 0.14 \\
\hline $\begin{array}{l}\mathrm{K}(\mathrm{mg} / 4184 \mathrm{~kJ} ; \text { median }) \\
\mathrm{BMI}\left(\mathrm{kg} / \mathrm{m}^{2}\right)\end{array}$ & \multicolumn{2}{|c|}{708} & \multicolumn{2}{|c|}{968} & \multicolumn{2}{|c|}{1198} & \multicolumn{2}{|c|}{1620} & \\
\hline Crude & $21 \cdot 0$ & 0.2 & 21.5 & 0.2 & $21 \cdot 3$ & 0.2 & $21 \cdot 1$ & 0.2 & 0.98 \\
\hline Adjusted $\ddagger$ & 21.3 & 0.2 & $21 \cdot 6$ & 0.2 & 21.3 & 0.2 & $20 \cdot 8$ & 0.2 & 0.03 \\
\hline \multicolumn{10}{|l|}{ Waist circumference $(\mathrm{cm})$} \\
\hline Crude & $72 \cdot 8$ & 0.4 & $73 \cdot 0$ & 0.4 & $73 \cdot 1$ & 0.4 & $72 \cdot 0$ & 0.4 & $0 \cdot 16$ \\
\hline Adjusted $\neq$ & 73.1 & 0.5 & $73 \cdot 0$ & 0.4 & $73 \cdot 1$ & 0.4 & 71.7 & 0.5 & 0.04 \\
\hline Protein ( $\%$ of energy; median) & \multicolumn{2}{|c|}{$10 \cdot 3$} & \multicolumn{2}{|c|}{$12 \cdot 6$} & \multicolumn{2}{|c|}{$14 \cdot 6$} & \multicolumn{2}{|c|}{$17 \cdot 9$} & \\
\hline BMI $\left(\mathrm{kg} / \mathrm{m}^{2}\right)$ & & & & & & & & & \\
\hline Crude & $20 \cdot 9$ & 0.2 & $21 \cdot 1$ & 0.2 & $21 \cdot 6$ & $0 \cdot 2$ & 21.4 & 0.2 & 0.005 \\
\hline Adjustedł & $20 \cdot 7$ & 0.2 & $21 \cdot 1$ & 0.2 & 21.5 & 0.2 & $21 \cdot 6$ & 0.2 & 0.0008 \\
\hline \multicolumn{10}{|l|}{ Waist circumference $(\mathrm{cm})$} \\
\hline Crude & 72.5 & 0.4 & $72 \cdot 7$ & 0.4 & $73 \cdot 0$ & 0.4 & $72 \cdot 7$ & 0.4 & 0.75 \\
\hline Adjusted $\ddagger$ & $71 \cdot 7$ & 0.5 & $72 \cdot 6$ & 0.4 & $73 \cdot 1$ & 0.4 & 73.5 & 0.5 & 0.02 \\
\hline \multicolumn{10}{|l|}{ Self-reported dietary intake§ } \\
\hline $\mathrm{Na}(\mathrm{mg} / 4184 \mathrm{~kJ} ;$ median) & & & & & & & & & \\
\hline BMI $\left(\mathrm{kg} / \mathrm{m}^{2}\right)$ & & & & & & & & & \\
\hline Crude & 21.4 & 0.2 & $21 \cdot 3$ & 0.2 & $21 \cdot 2$ & 0.2 & $21 \cdot 0$ & 0.2 & 0.11 \\
\hline Adjusted $\neq$ & $21 \cdot 2$ & 0.2 & $21 \cdot 2$ & 0.2 & 21.3 & 0.2 & $21 \cdot 2$ & 0.2 & 0.94 \\
\hline Waist circumference $(\mathrm{cm})$ & & & & & & & & & \\
\hline Crude & $73 \cdot 7$ & 0.4 & $72 \cdot 9$ & 0.4 & $72 \cdot 2$ & 0.4 & $72 \cdot 1$ & 0.4 & 0.004 \\
\hline Adjusted $\neq$ & 73.3 & 0.4 & $72 \cdot 8$ & 0.4 & 72.4 & 0.4 & $72 \cdot 4$ & 0.4 & 0.14 \\
\hline $\mathrm{K}$ (mg/4184 kJ; median) & & & & & & & & & \\
\hline Crude & $21 \cdot 7$ & 0.2 & $21 \cdot 2$ & 0.2 & $21 \cdot 3$ & 0.2 & $20 \cdot 7$ & 0.2 & $<0.0001$ \\
\hline Adjusted $\ddagger$ & 21.6 & 0.2 & $21 \cdot 2$ & 0.2 & 21.3 & 0.2 & $20 \cdot 8$ & 0.2 & 0.009 \\
\hline Waist circumference $(\mathrm{cm})$ & & & & & & & & & \\
\hline Crude & $74 \cdot 2$ & 0.4 & $72 \cdot 9$ & 0.4 & $72 \cdot 8$ & 0.4 & $71 \cdot 1$ & 0.4 & $<0.0001$ \\
\hline Adjusted $\ddagger$ & $73 \cdot 8$ & 0.5 & $72 \cdot 9$ & 0.4 & $72 \cdot 8$ & 0.4 & 71.4 & 0.5 & 0.001 \\
\hline Protein (\% of energy; median) & & & & & & & & & \\
\hline $\operatorname{BMI}\left(\mathrm{kg} / \mathrm{m}^{2}\right)$ & & & & & & & & & \\
\hline Crude & $21 \cdot 6$ & 0.2 & $21 \cdot 3$ & 0.2 & $21 \cdot 2$ & 0.2 & $20 \cdot 8$ & 0.2 & 0.0002 \\
\hline Adjusted $\ddagger$ & 21.4 & 0.2 & $21 \cdot 3$ & 0.2 & $21 \cdot 2$ & 0.2 & $20 \cdot 9$ & 0.2 & 0.08 \\
\hline Waist circumference $(\mathrm{cm})$ & & & & & & & & & \\
\hline Crude & 73.9 & 0.4 & $73 \cdot 0$ & 0.4 & 72.5 & 0.4 & 71.6 & 0.4 & $<0.0001$ \\
\hline Adjusted $\ddagger$ & $73 \cdot 2$ & 0.5 & $72 \cdot 8$ & 0.4 & $72 \cdot 7$ & 0.4 & $72 \cdot 3$ & 0.5 & 0.20 \\
\hline
\end{tabular}

${ }^{*}$ A linear trend test was used with the median value in each quartile category of dietary intake as a continuous variable in linear regression.

†Energy adjustment was made using estimated energy requirement.

$\ddagger$ Adjusted for survey year (2006 or 2007), region (north, central or south), municipality level (ward, city, or town and village), residential status (living with family, living alone or living with others), current alcohol drinking (yes or no), current smoking status (yes or no), physical activity (total metabolic equivalents-h/d, continuous), and $24 \mathrm{~h}$ urine-derived or self-reported intakes of the other two nutrients (continuous).

$\S$ Energy adjustment was made using self-reported energy intake.

When self-reported information was used to estimate dietary intakes, $\mathrm{Na}$ and protein intakes were not associated with both general and abdominal obesity. However, self-reported $\mathrm{K}$ intake was associated with a lower risk of both general (adjusted OR 0.39; $95 \%$ CI $0.17,0.92 ; P$ for trend=0.04) and abdominal (adjusted OR 0.48; 95\% CI: 0.24, 0.93; $P$ for trend=0.02) obesity.

\section{Discussion}

To our knowledge, this is the first study to investigate the ability of self-reported estimates of dietary intakes of $\mathrm{Na}, \mathrm{K}$ and protein to detect an association with obesity, by comparison with the corresponding estimates derived from $24 \mathrm{~h}$ urinary excretion. When $24 \mathrm{~h}$ urinary excretion was used for estimating dietary intakes, we found the positive associations of $\mathrm{Na}$ and protein intakes with both general and abdominal obesity and the inverse association of $\mathrm{K}$ with abdominal obesity in a group of young Japanese women. However, no association was observed based on using self-reported dietary intakes, except for inverse associations between $\mathrm{K}$ intake and general and abdominal obesity. These results suggest that the ability of self-reported information using the DHQ, at least for 
Table 5. Odds ratios for general and abdominal obesity according to the quartiles (Q) of sodium, potassium and protein intakes $(n 1043)^{\star}$ (Odds ratios and $95 \%$ confidence intervals)

\begin{tabular}{|c|c|c|c|c|c|c|c|c|}
\hline & \multirow{2}{*}{$\frac{\mathrm{Q} 1(n \mathrm{260})}{\text { OR (reference) }}$} & \multicolumn{2}{|c|}{ Q2 (n 261) } & \multicolumn{2}{|c|}{ Q3 (n 261) } & \multicolumn{2}{|c|}{ Q4 (n 261) } & \multirow[b]{2}{*}{$P$ for trend $\dagger$} \\
\hline & & OR & $95 \% \mathrm{Cl}$ & OR & $95 \% \mathrm{Cl}$ & OR & $95 \% \mathrm{Cl}$ & \\
\hline \multicolumn{9}{|l|}{$24 \mathrm{~h}$ Urine-derived dietary intake $\neq$} \\
\hline $\mathrm{Na}(\mathrm{mg} / 4184 \mathrm{~kJ} ;$ median) & 1155 & & 1659 & & 2124 & & 2766 & \\
\hline General obesity (\%) & $5 \cdot 0$ & & 8.4 & & 6.5 & & $10 \cdot 7$ & \\
\hline Crude model & 1 & 1.75 & $0.86,3.55$ & 1.32 & $0.63,2 \cdot 78$ & $2 \cdot 28$ & $1 \cdot 16,4.52$ & 0.03 \\
\hline Adjusted model§ & 1 & 1.81 & $0.88,3.74$ & 1.43 & $0.66,3.09$ & 2.49 & $1 \cdot 15,5 \cdot 42$ & 0.04 \\
\hline Abdominal obesity (\%) & 11.5 & & 11.5 & & $12 \cdot 3$ & & $16 \cdot 9$ & \\
\hline Crude model & 1 & 1.00 & $0.58,1.71$ & 1.07 & $0 \cdot 63,1 \cdot 82$ & 1.56 & $0.94,2.56$ & 0.06 \\
\hline Adjusted model§ & 1 & 1.06 & $0.61,1.83$ & $1 \cdot 20$ & $0.69,2.09$ & 1.77 & $1.00,3 \cdot 16$ & 0.04 \\
\hline $\mathrm{K}$ (mg/4184 kJ; median) & 708 & & 968 & & 1198 & & 1620 & \\
\hline General obesity (\%) & $6 \cdot 2$ & & $10 \cdot 0$ & & $8 \cdot 1$ & & $6 \cdot 5$ & \\
\hline Crude model & 1 & 1.69 & $0.88,3 \cdot 23$ & 1.33 & $0 \cdot 68,2 \cdot 62$ & 1.06 & $0.53,2.15$ & 0.80 \\
\hline Adjusted model§ & 1 & 1.48 & $0.75,2.90$ & 1.03 & $0.49,2 \cdot 16$ & 0.60 & $0.26,1.40$ & 0.09 \\
\hline Abdominal obesity (\%) & $14 \cdot 6$ & & $13 \cdot 8$ & & 11.9 & & 11.9 & \\
\hline Crude model & 1 & 0.94 & $0.57,1.53$ & 0.79 & $0.47,1.31$ & 0.79 & $0.47,1.31$ & 0.30 \\
\hline Adjusted model§ & 1 & 0.78 & $0.47,1.31$ & 0.59 & $0.33,1.04$ & 0.46 & $0.25,0.87$ & 0.01 \\
\hline Protein (\% of energy; median) & $10 \cdot 3$ & & $12 \cdot 6$ & & $14 \cdot 6$ & & $17 \cdot 9$ & \\
\hline General obesity (\%) & 4.6 & & $7 \cdot 7$ & & 9.6 & & 8.8 & \\
\hline Crude model & 1 & 1.72 & $0.82,3.59$ & $2 \cdot 19$ & $1.08,4.46$ & $2 \cdot 00$ & $0.97,4 \cdot 10$ & 0.07 \\
\hline Adjusted model§ & 1 & 2.02 & $0.94,4.36$ & $2 \cdot 71$ & $1 \cdot 24,5 \cdot 94$ & 2.95 & $1 \cdot 21,7 \cdot 22$ & 0.02 \\
\hline Abdominal obesity (\%) & $11 \cdot 2$ & & $13 \cdot 0$ & & $13 \cdot 8$ & & 14.2 & \\
\hline Crude model & 1 & $1 \cdot 19$ & $0.70,2.02$ & $1 \cdot 27$ & $0 \cdot 76,2 \cdot 15$ & 1.32 & $0 \cdot 78,2 \cdot 21$ & 0.31 \\
\hline Adjusted model§ & 1 & 1.46 & $0.84,2.54$ & 1.55 & $0 \cdot 87,2 \cdot 77$ & 1.97 & $1.02,3.83$ & 0.053 \\
\hline \multicolumn{9}{|l|}{ Self-reported dietary intake\| } \\
\hline $\mathrm{Na}(\mathrm{mg} / 4184 \mathrm{~kJ} ;$ median) & 1526 & & 1882 & & 2167 & & 2580 & \\
\hline General obesity (\%) & 8.5 & & 8.4 & & $5 \cdot 8$ & & $8 \cdot 1$ & \\
\hline Crude model & 1 & 1.00 & $0.54,1.85$ & 0.66 & $0.33,1.30$ & 0.95 & $0.51,1.77$ & 0.66 \\
\hline Adjusted model§ & 1 & 1.13 & $0.59,2 \cdot 14$ & 0.85 & $0.41,1.74$ & 1.23 & $0.61,2.47$ & 0.70 \\
\hline Abdominal obesity (\%) & $17 \cdot 7$ & & $14 \cdot 2$ & & $9 \cdot 6$ & & $10 \cdot 7$ & \\
\hline Crude model & 1 & 0.77 & $0.48,1.23$ & 0.49 & $0.29,0.83$ & 0.60 & $0.34,0.93$ & 0.009 \\
\hline Adjusted model§ & 1 & 0.84 & $0.51,1.38$ & 0.60 & $0.34,1.03$ & 0.77 & $0.44,1.36$ & 0.22 \\
\hline $\mathrm{K}$ (mg/4184 kJ; median) & 837 & & 1015 & & 1173 & & 1393 & \\
\hline General obesity (\%) & $10 \cdot 8$ & & $8 \cdot 1$ & & $7 \cdot 3$ & & 4.6 & \\
\hline Crude model & 1 & 0.73 & $0.40,1.31$ & 0.65 & $0.35,1 \cdot 20$ & 0.40 & $0.20,0.80$ & 0.01 \\
\hline Adjusted model§ & 1 & 0.71 & $0.38,1.33$ & 0.67 & $0.34,1.34$ & 0.39 & $0.17,0.92$ & 0.04 \\
\hline Abdominal obesity (\%) & $19 \cdot 2$ & & $14 \cdot 2$ & & $11 \cdot 1$ & & $7 \cdot 7$ & \\
\hline Crude model & 1 & 0.69 & $0.44,1 \cdot 10$ & 0.53 & $0.32,0.86$ & 0.35 & $0.20,0.60$ & $<0.0001$ \\
\hline Adjusted model§ & 1 & 0.79 & $0.48,1.29$ & 0.62 & $0.36,1.07$ & 0.48 & $0.24,0.93$ & 0.02 \\
\hline Protein (\% of energy; median) & 11.5 & & $12 \cdot 9$ & & $14 \cdot 0$ & & $15 \cdot 6$ & \\
\hline General obesity (\%) & $10 \cdot 8$ & & $8 \cdot 1$ & & $5 \cdot 8$ & & $6 \cdot 1$ & \\
\hline Crude model & 1 & 0.73 & $0.40,1.31$ & 0.51 & $0.26,0.97$ & 0.54 & $0.29,1.03$ & 0.03 \\
\hline Adjusted model§ & 1 & 0.72 & $0.38,1.36$ & 0.47 & $0.23,0.99$ & 0.53 & $0 \cdot 23,1 \cdot 22$ & 0.09 \\
\hline Abdominal obesity (\%) & $18 \cdot 9$ & & 14.9 & & $9 \cdot 2$ & & $9 \cdot 2$ & \\
\hline Crude model & 1 & 0.76 & $0.48,1.20$ & 0.44 & $0.26,0.74$ & 0.44 & $0.26,0.74$ & 0.0003 \\
\hline Adjusted model§ & 1 & 0.86 & $0.52,1.41$ & 0.54 & $0.30,0.98$ & 0.64 & $0.33,1.27$ & 0.11 \\
\hline
\end{tabular}

*General obesity was defined as $\mathrm{BMI} \geq 25 \mathrm{~kg} / \mathrm{m}^{2}$; abdominal obesity was defined as waist circumference $\geq 80 \mathrm{~cm}{ }^{(27)}$.

† Logistic regression models were used with the median value in each quartile category of dietary intake as a continuous variable in logistic regression.

† Energy adjustment was made using estimated energy requirement.

$\S$ Adjusted for survey year (2006 or 2007), region (north, central or south), municipality level (ward, city, or town and village), residential status (living with family, living alone or living with others), current alcohol drinking (yes or no), current smoking status (yes or no), physical activity (total metabolic equivalents-h/d, continuous) and $24 \mathrm{~h}$ urine-derived or self-reported intakes of other two nutrients (continuous).

\| Energy adjustment was made using self-reported energy intake.

estimating $\mathrm{Na}$ and protein intakes in terms of investigating diet-obesity relationships, is limited.

Only a limited number of studies have investigated the dietary intakes of protein, $\mathrm{Na}$ and $\mathrm{K}$ in relation to body fatness. Self-reported protein intake was positively associated with subsequent weight gain in two very large prospective studies in European countries ${ }^{(34,35)}$. Very recently, a small study in middle-aged Danish men and women has shown that both self-reported and $24 \mathrm{~h}$ urine-derived protein intakes are associated with greater weight gain ${ }^{(13)}$. Consistent with these studies, we found positive associations between $24 \mathrm{~h}$ urinederived protein intake and general and abdominal obesity. For $\mathrm{Na}$, self-reported intake was cross-sectionally associated with a higher risk of obesity in Korean children and adults, independent of energy intake ${ }^{(14)}$. The $24 \mathrm{~h}$ urinary excretion was cross-sectionally associated with higher BMI in young Swedish men ${ }^{(15)}$ and higher BMI and WC in middle-aged men and women in Venezuela ${ }^{(16)}$, although no adjustment was made in these two studies. In a study in German children and adolescents, higher $24 \mathrm{~h} \mathrm{Na}$ excretion was associated with 
subsequent increase in body fat percentage after adjustment for sugar-sweetened beverages or energy intake ${ }^{(17)}$. In middle-aged Danish men and women, $24 \mathrm{~h}$ urinary excretion of $\mathrm{Na}$ was associated positively with change in fat mass and inversely with change in fat-free mass, independently of energy intake ${ }^{(18)}$. We also found that $24 \mathrm{~h}$ urine-derived $\mathrm{Na}$ intake was associated with a higher risk of general and abdominal obesity with energy adjustment. For K, we are not aware of studies where $24 \mathrm{~h}$ urinary $\mathrm{K}$ is investigated in relation to body fatness; however, self-reported $\mathrm{K}$ intake was inversely associated with abdominal obesity independent of energy and macronutrient intakes in a Korean national representative cross-sectional study ${ }^{(19)}$. Additionally, the ratio of urinary $\mathrm{Na}$ to $\mathrm{K}$ in a first-void morning urinary sample was cross-sectionally associated with lower percentage body fat in a multiethnic cohort in the USA ${ }^{(20)}$. We also found inverse associations between $24 \mathrm{~h}$ urine-derived and self-reported $\mathrm{K}$ intake and general (self-report only) and abdominal obesity, although the present study found no association between the ratio of $\mathrm{Na}$ to $\mathrm{K}$ and obesity (data not shown).

While all the three nutrients were associated with obesity when dietary intake was derived from $24 \mathrm{~h}$ urinary excretion, only $\mathrm{K}$, but not $\mathrm{Na}$ and protein, was associated with obesity when self-reported dietary intake was used. This seems reasonable given the modest (although still low) correlation between $24 \mathrm{~h}$ urine-derived and self-reported intakes for $\mathrm{K}$ compared with very low correlations for $\mathrm{Na}$ and protein. Additionally, as the validity of the DHQ has been examined mainly in adults ${ }^{(29-32)}$, the validity of the instrument for assessing dietary intakes in young women is largely unknown. Further, the DHQ is not suitable for assessing Na intakes ${ }^{(29)}$, as are other dietary assessment questionnaires. In any case, the present findings highlight the difficulty in obtaining valid estimates of dietary intake (at least of some nutrients such as $\mathrm{Na}$ and protein) from self-reported dietary information using the DHQ as a basis for investigating diet-obesity relationships.

The strengths of the present study include the use of objective biomarkers for dietary intakes of $\mathrm{Na}, \mathrm{K}$ and protein and measured anthropometric data in a relatively large sample. However, there are also several limitations. First, the crosssectional nature of the study does not permit the assessment of causality owing to the uncertain temporality of the association. Only a prospective study with the use of biomarkers or carefully taking into account dietary misreporting would provide better understanding of the relationship between dietary intakes of $\mathrm{Na}, \mathrm{K}$ and protein and obesity. Second, the present results were based on a highly selected population of female dietetic students, who probably had a higher education level and greater knowledge of diet and nutrition than the general population. It is not clear how such characteristics affected the associations we observed here. Thus, the present findings may only be specific to the present population and may not be extrapolatable to the Japanese population in general. Third, obtaining a valid dietary intake derived from urine excretion requires that $24 \mathrm{~h}$ urine collection be complete. We, therefore, excluded participants whose $24 \mathrm{~h}$ urine collection was considered incomplete, as assessed based on a strategy ${ }^{(26)}$ validated against the PABA check method ${ }^{(24)}$, as described above. Also, a repeated analysis in 601 women having complete urine assessed by PABA (PABA recovery $\geq 85 \%$ ) in a subsample ( $n$ 654) of the present participants provided similar results (data not shown). Although our use of only a single $24 \mathrm{~h}$ urine sample is not an optimal way to characterise individual habitual dietary intake and introduces random errors $^{(36)}$, this kind of error would nevertheless tend to result in bias towards attenuating rather than enhancing the relationship, and multiple $24 \mathrm{~h}$ urine collections would have only provided more precise results. Fourth, concerns have been expressed regarding the precision of the correction factors used to estimate the dietary intakes from $24 \mathrm{~h}$ urine. Many factors may influence the percentage of dietary protein $(\mathrm{N})$, $\mathrm{K}$ and $\mathrm{Na}$ excreted in the urine, including the absolute level of dietary intake, season during which the balance studies is conducted, race and cooking methods ${ }^{(37)}$. Here, we used correction factors observed in previous carefully designed balance studies ${ }^{(9-11)}$. Fifth, $24 \mathrm{~h}$ urine-derived dietary intake was energy-adjusted using estimated energy requirement calculated based on an equation from the US Dietary Reference intakes ${ }^{(28)}$ in addition to non-validated information on self-reported physical activity. Although the equation was developed based on a large number of measurements of total energy expenditure by the doubly labelled water method and is highly accurate, it is predominantly based on data from white populations ${ }^{(28)}$, and might, therefore, be inappropriate for the present Japanese population. Nonetheless, as we found the similar results when the associations between $24 \mathrm{~h}$ urine-derived intakes and obesity were examined without energy-adjustment (data not shown), any measurement error of estimated energy requirement would not have a major impact on the present findings. Sixth, self-reported dietary data were collected using a dietary assessment questionnaire (i.e. DHQ). Thus, the present findings might be specific to this dietary assessment questionnaire and should be interpreted in this context, and different dietary assessment methods may perform differently. It should also be emphasised that the DHQ and a $24 \mathrm{~h}$ urinary collection assess dietary intake over different time periods, namely in the previous month for the former and in the previous $1 \mathrm{~d}$ for the latter. Ideally, the associations between obesity and self-reported and biomarker-based intakes should be investigated over similar time frames for comparing them. Finally, although we adjusted for a variety of potential confounding variables, residual confounding could not be ruled out.

In conclusion, in this cross-sectional study in a group of young Japanese women, we showed positive associations between $\mathrm{Na}$ and protein intakes and general and abdominal obesity, and an inverse association between $\mathrm{K}$ intake and abdominal obesity when dietary intake was estimated on the basis of $24 \mathrm{~h}$ urinary excretion. Conversely, when self-reported information was used to estimate dietary intake, only associations between $\mathrm{K}$ and general and abdominal obesity were observed, with no association for $\mathrm{Na}$ and protein intakes, suggesting only limited ability of self-reported dietary intakes of, at least, $\mathrm{Na}$ and protein using the DHQ to detect diet-obesity associations. The present study highlights the need to critically evaluate self-reported dietary data in diet-obesity research as well as potential usefulness 
of even a single $24 \mathrm{~h}$ urine collection. Further research using a self-reported instrument and a biomarker with the same reference period (e.g. multiple $24 \mathrm{~h}$ recalls and multiple $24 \mathrm{~h}$ urinary collections) would be of interest.

\section{Acknowledgements}

The members of the Japan Dietetic Students' Study for Nutrition and Biomarkers Group (in addition to the authors) are as follows (affiliation at the time of data collection in parentheses): Mitsuyo Yamasaki, Yuko Hisatomi, Junko Soezima, and Kazumi Takedomi (Nishikyushu University); Toshiyuki Kohri and Naoko Kaba (Kinki University); Etsuko Uneoka (Otemae College of Nutrition); Hitomi Hayabuchi and Yoko Umeki (Fukuoka Women's University); Keiko Baba and Maiko Suzuki (Mie Chukyo University Junior College); Reiko Watanabe and Kanako Muramatsu (Niigata Women's College); Kazuko Ohki, Seigo Shiga, Hidemichi Ebisawa, and Masako Fuwa (Showa Women's University); Tomoko Watanabe, Ayuho Suzuki, and Fumiyo Kudo (Chiba College of Health Science); Katsumi Shibata, Tsutomu Fukuwatari, and Junko Hirose (The University of Shiga Prefecture); Toru Takahashi and Masako Kato (Mimasaka University); Toshinao Goda and Yoko Ichikawa (University of Shizuoka); Junko Suzuki, Yoko Niida, Satomi Morohashi, Chiaki Shimizu, and Naomi Takeuchi (Hokkaido Bunkyo University); Jun Oka and Tomoko Ide (Tokyo Kasei University); and Yoshiko Sugiyama and Mika Furuki (Minamikyushu University).

The present study was supported, in part, by grants from the Ministry of Health, Labour, and Welfare of Japan (S. S., grant numbers 200400526A, 200500555A, 200624013A and 200624013B). The Ministry of Health, Labour, and Welfare of Japan had no role in the design and analysis of the present study or in the writing of this article.

The authors' contributions are as follows: K. M. contributed to the concept and design of the survey, coordination of the fieldwork, data collection and management, hypothesis formulation, statistical analysis, data interpretation and manuscript writing; M. B. E. L. helped in writing of the manuscript; S. S. contributed to the concept and design of the survey, data collection and manuscript editing; K. U. contributed to the concept and design of the survey and data collection. All authors read and approved the final manuscript.

None of the authors has any conflict of interest.

\section{References}

1. Livingstone MBE \& Black AE (2003) Markers of the validity of reported energy intake. J Nutr 133, Suppl. 3, 895S-920S

2. Subar AF, Kipnis V, Troiano RP, et al. (2003) Using intake biomarkers to evaluate the extent of dietary misreporting in a large sample of adults: the OPEN study. Am J Epidemiol 158, $1-13$.

3. Tooze JA, Subar AF, Thompson FE, et al. (2004) Psychosocial predictors of energy underreporting in a large doubly labeled water study. Am J Clin Nutr 79, 795-804.

4. Rosell MS, Hellenius MLB, De Faire UH, et al. (2003) Associations between diet and the metabolic syndrome vary with the validity of dietary intake data. Am J Clin Nutr 78, 84-90.
5. Mattisson I, Wirfalt E, Aronsson CA, et al. (2005) Misreporting of energy: prevalence, characteristics of misreporters and influence on observed risk estimates in the Malmo Diet and Cancer cohort. Br J Nutr 94, 832-842.

6. Heitmann BL \& Lissner L (1995) Dietary underreporting by obese individuals: is it specific or non-specific? BMJ 311, 986-989.

7. Goris AHC, Westerterp-Plantenga MS \& Westerterp KR (2000) Undereating and underrecording of habitual food intake in obese men: selective underreporting of fat intake. Am J Clin Nutr 71, 130-134.

8. Poppitt SD, Swann D, Black AE, et al. (1998) Assessment of selective under-reporting of food intake by both obese and non-obese women in a metabolic facility. Int $J$ Obes Relat Metab Disord 22, 303-311.

9. Bingham SA \& Cummings JH (1985) Urine nitrogen as an independent validatory measure of dietary intake: a study of nitrogen balance in individuals consuming their normal diet. Am J Clin Nutr 42, 1276-1289.

10. Holbrook JT, Patterson KY, Bodner JE, et al. (1984) Sodium and potassium intake and balance in adults consuming self-selected diets. Am J Clin Nutr 40, 786-793.

11. Tasevska N, Runswick SA \& Bingham SA (2006) Urinary potassium is as reliable as urinary nitrogen for use as a recovery biomarker in dietary studies of free living individuals. $J$ Nutr 136, 1334-1340.

12. Michaelsen KF \& Greer FR (2014) Protein needs early in life and long-term health. Am J Clin Nutr 99, 718S-722S.

13. Ankarfeldt MZ, Gottliebsen K, Angquist L, et al. (2015) Dietary protein and urinary nitrogen in relation to 6-year changes in fat mass and fat-free mass. Int J Obes (Lond) 39, 162-168.

14. Yoon YS \& Oh SW (2013) Sodium density and obesity; the Korea National Health and Nutrition Examination Survey 2007-2010. Eur J Clin Nutr 67, 141-146.

15. Hulthen L, Aurell M, Klingberg S, et al. (2010) Salt intake in young Swedish men. Public Health Nutr 13, 601-605.

16. Hoffmann IS \& Cubeddu LX (2009) Salt and the metabolic syndrome. Nutr Metab Cardiovasc Dis 19, 123-128.

17. Libuda L, Kersting M \& Alexy U (2012) Consumption of dietary salt measured by urinary sodium excretion and its association with body weight status in healthy children and adolescents. Public Health Nutr 15, 433-441.

18. Larsen SC, Angquist L, Sorensen TI, et al. (2013) $24 \mathrm{~h}$ Urinary sodium excretion and subsequent change in weight, waist circumference and body composition. PLOS ONE 8, e69689.

19. Shin D, Joh HK, Kim KH, et al. (2013) Benefits of potassium intake on metabolic syndrome: The Fourth Korean National Health and Nutrition Examination Survey (KNHANES IV). Atherosclerosis 230, 80-85.

20. Jain N, Minhajuddin AT, Neeland IJ, et al. (2014) Association of urinary sodium-to-potassium ratio with obesity in a multiethnic cohort. Am J Clin Nutr 99, 992-998.

21. Fonseca-Alaniz MH, Brito LC, Borges-Silva CN, et al. (2007) High dietary sodium intake increases white adipose tissue mass and plasma leptin in rats. Obesity 15, 2200-2208.

22. Murakami K, Sasaki S \& Uenishi K (2012) The degree of misreporting of the energy-adjusted intake of protein, potassium, and sodium does not differ among under-, acceptable, and over-reporters of energy intake. Nutr Res 32, 741-750.

23. Vergnaud AC, Norat T, Romaguera D, et al. (2012) Fruit and vegetable consumption and prospective weight change in participants of the European Prospective Investigation into Cancer and Nutrition-Physical Activity, Nutrition, Alcohol, Cessation of Smoking, Eating Out of Home, and Obesity study. Am J Clin Nutr 95, 184-193. 
24. Murakami K, Sasaki S, Takahashi Y, et al. (2008) Sensitivity and specificity of published strategies using urinary creatinine to identify incomplete 24-h urine collection. Nutrition 24, 16-22.

25. Murakami K, Sasaki S, Takahashi Y, et al. (2008) Misreporting of dietary energy, protein, potassium and sodium in relation to body mass index in young Japanese women. Eur J Clin Nutr 62, 111-118.

26. Knuiman JT, Hautvast JG, van der Heyden L, et al. (1986) A multi-centre study on completeness of urine collection in 11 European centres. I. Some problems with the use of creatinine and 4-aminobenzoic acid as markers of the completeness of collection. Hum Nutr Clin Nutr 40, 229-237.

27. World Health Organization Western Pacific Region \& International Association for the Study of Obesity (2000) For the Study of Obesity Task Force: The Asia-Pacific Perspective: Redefining Obesity and its Treatment. Sydney: Health Communications Australia pp. 15-21.

28. Institute of Medicine (2002) Dietary Reference Intakes for Energy, Carbohydrate, Fiber, Fat, Fatty Acids, Cholesterol, Protein and Amino Acids. Washington, DC: National Academy Press.

29. Sasaki S, Yanagibori R \& Amano K (1998) Validity of a selfadministered diet history questionnaire for assessment of sodium and potassium: comparison with single 24-hour urinary excretion. Jpn Circ J 62, 431-435.

30. Okubo H, Sasaki S, Rafamantanantsoa HH, et al. (2008) Validation of self-reported energy intake by a self-administered diet history questionnaire using the doubly labeled water method in 140 Japanese adults. Eur J Clin Nutr 62, 1343-1350.

31. Kobayashi S, Honda S, Murakami K, et al. (2012) Both comprehensive and brief self-administered diet history questionnaires satisfactorily rank nutrient intakes in Japanese adults. J Epidemiol 22, 151-159.

32. Murakami K, Sasaki S, Takahashi Y, et al. (2008) Reproducibility and relative validity of dietary glycaemic index and load assessed with a self-administered diet-history questionnaire in Japanese adults. Br J Nutr 99, 639-648.

33. Science and Technology Agency (2005) Standard Tables of Food Composition in Japan, 5th revised and enlarged ed. Tokyo: Printing Bureau of the Ministry of Finance (in Japanese).

34. Halkjaer J, Olsen A, Overvad K, et al. (2011) Intake of total, animal and plant protein and subsequent changes in weight or waist circumference in European men and women: the Diogenes project. Int J Obes 35, 1104-1113.

35. Vergnaud AC, Norat T, Mouw T, et al. (2013) Macronutrient composition of the diet and prospective weight change in participants of the EPIC-PANACEA study. PLOS ONE $\mathbf{8}$, e57300.

36. Bingham SA (2003) Urine nitrogen as a biomarker for the validation of dietary protein intake. J Nutr 133, Suppl. 3, 921S-924S

37. Zhang J, Temme EHM, Sasaki S, et al. (2000) Under- and overreporting of energy intake using urinary cations as biomarkers: relation to body mass index. Am J Epidemiol 152, 453-462. 